\title{
Blood Urea Nitrogen as a Predictor of In-Hospital Mortality in Acute Coronary Syndrome Patients
}

\author{
Trisulo Wasyanto ${ }^{1,2)}$, Annisa Tridamayanti,1,2) \\ 1)Department of Cardiology and Vascular Medicine, Faculty of Medicine, \\ Universitas Sebelas Maret \\ 2)Dr. Moewardi Hospital, Surakarta
}

\begin{abstract}
Background: Acute coronary syndrome (ACS) is one of the major causes of morbidity and mortality worldwide. Thus, it is important to effectively diagnose and determine the prognosis and mortality risk. While criteria such as Thrombolysis in Myocardial Infarction (TIMI) and Global Registry of Acute Coronary Events (GRACE) score are clinically used to work out the prognosis of patients with ACS, the examination of Blood Urea Nitrogen (BUN) and creatinine alongside in predicting outcome may prove favorable as well. This study aimed to determine the efficacy of BUN and creatinine in mortality risk assessment of patients with ACS, and to find which one is better.

Subjects and Method: This was an analytical study with a cohort retrospective design included 1463 ACS patients in Dr. Moewardi hospital from January 2014 to July 2018. The relationship between admission BUN, creatinine level, and in-hospital mortality were analyzed with chi-square and logistic regression. Receiver Operating Characteristic (ROC) curve to determine which one better as a predictor of in-hospital mortality.

Results: The mean age of patients was 60 years old (mean $=60.08 ; \mathrm{SD}=11.04$ ), which $72.9 \%$ were men. From all sample, 232 (15.9\%) patients were died. In binary log regression models, elevated BUN ( $>50 \mathrm{mg} / \mathrm{dL})$ at admission was an independent predictor of in-hospital mortality $(\mathrm{OR}=4.01$; $95 \% \mathrm{CI}=1.0$ to $7.0 ; \mathrm{p}=0.001)$. Similar results were obtained for elevated creatinine $(>1.3 \mathrm{mg} / \mathrm{dL})$ at admission $(\mathrm{OR}=3.6 ; 95 \% \mathrm{CI}=2.2$ to $5.8 ; \mathrm{p}=0.031)$. ROC curves showed that area under the curve (AUC) of BUN (o.87) was higher than AUC of creatinine (o.61).

Conclusion: Elevated BUN and creatinine are independent predictors of in-hospital mortality in ACS patients. A high level BUN at admission is a more accurate predictor of in-hospital mortality than creatinine.
\end{abstract}

Keywords: blood urea nitrogen, creatinine, acute coronary syndrome

\section{Correspondence:}

Trisulo Wasyanto. Department of Cardiology and Vascular Medicine, Faculty of Medicine, Universitas Sebelas Maret/Dr. Moewardi Hospital. Jl. Kol. Sutarto 132, Surakarta 57126. Indonesia. Email: trisulo.wasyanto@gmail.com.

\section{BACKGROUND}

Acute Coronary Syndrome (ACS) is a leading cause of hospitalization and death in the majority of countries, especially in developing countries (Go AS et al,. 2013). The prevalence and incidence of ACS is also very high in Indonesians. The JAC (Jakarta Acute Coronary Syndrome) registry study concluded that nearly $11 \%$ of the population in Jakarta had ACS, with 4\% of them being ST Elevation Myocardial Infarction
(STEMI) (Dharma et al., 2016).The profile of patients with ACS is quite varied, so risk stratification is important to provide optimal care in choosing an intervention or medically treatment. Several models of risk stratification have been developed for ACS patients, several of them are Global Registry of Acute Coronary Events (GRACE), Thrombolysis in Myocardial Infarction (TIMI), and HEART score. This stratification can be done when the patient first 
admitted to hospital. These models consist of important prognostic factors, including age, gender, angina, risk factors for coronary artery disease (CAD), signs of heart failure, systolic blood pressure, heart rate, cardiac arrest during admission, ST segment changes, cardiac enzymes, and serum creatinine. Increased serum creatinine levels, which are commonly used as markers of kidney function, are also known as predictors of ACS outcome. According to Granger and friends, creatinine increase of $1.0 \mathrm{mg} / \mathrm{dL}$ is increasing the risk of death in ACS patients by $15-35 \%$ (Granger et al., 2003).

Creatinine as a marker of kidney function is predictors for worse outcomes in ACS patients (Shiraishi et al., 2016). Estimation of Glomerulus Filtration Rate (GFR), which is calculated by serum creatinine, is also a marker of kidney function. A reduction in the estimated GFR has also been reported as a predictor for poor prognosis in ACS patients (Lee et al., 2016). However, kidney function based on serum creatinine levels may not perfectly reflect the actual GFR, because serum creatinine levels are influenced by several factors, such as age, sex, muscle mass, and diet (Kimura et al., 2016). Blood Urea Nitrogen (BUN) is a marker of other kidney functions. The urea concentration is determined by the balance of excretion and reabsorption in the kidney. Urea reabsorption is closely related to water reabsorption in the distal nephron which is under the influence of antidiuretic hormones whose excretion is affected by angiotensin II (Aronson et al., 2004). In addition to low GFR conditions, increased urea levels are associated with activation of the renin-angiotensin-aldosterone system and sympathetic nerves, which may be caused by renal hypoperfusion from hypovolemia, renovascular disease, and decreased cardiac output
(Kazory et al., 2010). Therefore, urea can be a marker that reflects disorders of cardio renal function and neurohormonal activetion. In patients with heart failure, high urea levels are a marker of strong mortality compared to creatinine (Chen et al., 2012). This relationship has also been shown in patients with ACS (Gibson et al., 2003).

In developing country populations, especially Indonesia, the relationship between BUN and mortality in ACS patients has not been widely described by researchers. There is a possibility that BUN is a better predictor of mortality compared to creatinine, seeing that several studies in developing countries have proven its significance (Grundy et al., 2018).

\section{SUBJECTS AND METHOD}

This study was an analytical study with a cohort retrospective design to determine the relationship between BUN and creatinine with hospital mortality. Patient sample data and results of laboratory tests was taken from the medical records installation of Dr. Moewardi Hospital from January 2014 to July 2018 that were diagnosed with STEMI, Non ST Elevation Myocardial Infarction (NSTEMI) and Unstable Angina Pectoris (UAP) based on clinical, electrocardiographic features and laboratory examinations. Exclusion criteria are patients with incomplete data, history of chronic kidney disease and hemodialysis. The independent variables for cohort analysis in this study were age, gender, risk factors (diabetes mellitus, hypertension, previous coronary heart disease and smoking), hemoglobin, leukocytes, platelets, urea, creatinine, sodium, potassium, chloride, and glucose. The dependent variable in this study was mortality during hospitalization.

Acute myocardial infarction according to European Society of Cardiology (ESC) in Fourth Universal Definition of Myocardial 
Infarction (Thygesen et al., 2019) is defined as detection of an increase and/or decrease in cardiac biomarkers with at least 1 value above the $99^{\text {th }}$ percentile of the upper reference limit, together with evidence of myocardial ischemia with at least one of the following: symptoms of ischemia, changes in electrocardiography (ECG) that indicate new ischemia and evolution of pathological $\mathrm{Q}$ waves on the ECG, and imaging evidence decreases new myocardial viability or abnormal segmental motion of the myocardial wall. Hypertension is defined as an increase in systolic blood pressure $>140 \mathrm{mmHg}$ and diastolic $>90$ at least two measurements with or without the use of antihypertensive drugs (Grundy et al., 2018). Diabetes mellitus (DM) is defined if fasting blood sugar $>126 \mathrm{mg} / \mathrm{dL}, 2$ hours post prandial $>200 \mathrm{mg} / \mathrm{dL}, \mathrm{HbA1C}>6.5$, and with or without the use of antidiabetic drugs (American Diabetes Association, 2004). Smoking is defined as a history of smoking within 10 years. Dyslipidemia is stated when LDL $>100 \mathrm{mg} / \mathrm{dL}$ (Catapano et al., 2016). Inhospital mortality is stated as a death that occurs during hospitalization.

Continuous data are presented as mean \pm SD if distributed normally, while distributed abnormally are presented as median (min-max). Categorical variables are expressed as a percentage (\%). The baseline characteristics of patients in the study group were compared by independent sample t-test for continous distributed normally variables, and Mann-Whitney for continuous variable that was abnormally distributed. For categorical variables we analyzed with $\mathrm{X}^{2}$. The relationship BUN and creatinine with hospital mortality was analyzed with bivariate $X^{2}$ test. Based on bivariate analysis, variables related to hospital mortality with $\mathrm{p}<0.05$ were included in multivariate analysis using logistic regression analysis. Receiver Operating Characteristic (ROC) was used to see the level of specificity and sensitivity of urea and creatinine and determine between the two which were better as predictors of hospital mortality. The results of the study were expressed as $\mathrm{p}$ values, Area Under Curve (AUC), Odd Ratio (OR) and 95\% Confident Intervals (CI), with $\mathrm{p}<0.050$ was considered significant.

\begin{tabular}{lcll}
\hline \multicolumn{4}{c}{ RESULTS } \\
\hline Clinical & Characteristic & of & ACS \\
Patients & & &
\end{tabular}

A total of 1680 patients with Acute Coronary Syndrome (ACS) were included in this study. Of these patients, 38 patients were excluded because had history of Chronic Kidney Disease (CKD) or history of hemodialysis, 217 patients were excluded because of incomplete data. We analyzed a total of 1425 samples of patients with ACS, 1062 (73.9\%) of whom were male and 363 (26.1\%) were women. The average age in the subjects of this study was $60.08 \pm 11.04$ years, with the youngest age was 23 years, and the oldest age was 97 years. Of the total ACS events, 909 (62.1\%) cases were STEMI, 296 (20.4\%) cases were NSTEMI, and 256 (17.5\%) were UAP

In the sample population of men who experienced ACS, 65.7\% were diagnosed with STEMI, while in the female group, fewer were diagnosed with STEMI than men, namely $55.6 \%$, the rest were diagnosed as NSTEMI (22.3\%) and UAP (22.1\%). Patients with complications of heart failure are characterized by Killip classification. In this study, there were 1026 patients (72.7\%) with Killip I, and 399 patients (27.3\%) Killip $\geq$ II, of which $74.4 \%$ of patients with Killip $\geq$ II were STEMI patients (Table 1). 
Indonesian Journal of Medicine (2019), 4(3): 241-251

https://doi.org/10.26911/theijmed.2019.04.03.07

Table 1. Risk Factor of patients in relation to type of ACS

\begin{tabular}{llllll}
\hline & & \multicolumn{3}{c}{ Acute Coronary Syndrome } \\
\cline { 3 - 6 } & & \multicolumn{1}{c}{ Total } & \multicolumn{1}{c}{ STEMI } & \multicolumn{1}{c}{ NSTEMI } & \multicolumn{1}{c}{ UAP } \\
\hline Gender & Male & $1062(73.9 \%)$ & $711(65.7 \%)$ & $209(19.3 \%)$ & $162(14.9 \%)$ \\
& Female & $363(26.1 \%)$ & $212(55.6 \%)$ & $85(22.3 \%)$ & $84(22.1 \%)$ \\
Killip & Killip I & $1026(72.7 \%)$ & $626(58.8 \%)$ & $210(19.7 \%)$ & $228(21.4 \%)$ \\
& Killip $\geq$ II & $399(27.3 \%)$ & $297(74.4 \%)$ & $84(21.1 \%)$ & $18(4.5 \%)$ \\
Hypertension & Yes & $725(50.9 \%)$ & $441(59.1 \%)$ & $166(22.3 \%)$ & $139(18.6 \%)$ \\
& No & $699(49.1 \%)$ & $482(67.2 \%)$ & $128(17.8 \%)$ & $107(14.9 \%)$ \\
Diabetes & Yes & $383(26.9 \%)$ & $255(64.5 \%)$ & $75(18.9 \%)$ & $65(16.6 \%)$ \\
Mellitus & No & $1042(73.1 \%)$ & $668(62.5 \%)$ & $219(20.5 \%)$ & $181(17.0 \%)$ \\
History & Yes & $171(12.1 \%)$ & $61(34.2 \%)$ & $60(33.7 \%)$ & $57(32.1 \%)$ \\
CAD & No & $1254(87.9 \%)$ & $861(67.0 \%)$ & $234(18.2 \%)$ & $189(14.7 \%)$ \\
Smoking & Yes & $845(59.3 \%)$ & $581(67.1 \%)$ & $156(17.9 \%)$ & $130(15.0 \%)$ \\
& No & $580(40.7 \%)$ & $342(57.4 \%)$ & $138(23.1 \%)$ & $116(19.4 \%)$ \\
Dyslipidemia & Yes & $299(21.3 \%)$ & $161(52.1 \%)$ & $102(32.8 \%)$ & $48(15.1 \%)$ \\
& No & $1126(78.7 \%)$ & $762(60.9 \%)$ & $192(19.3 \%)$ & $198(19.8 \%)$ \\
\hline
\end{tabular}

The most prevalent cardiovascular disease risk factors for patients with ACS in Dr. Moewardi hospital is smoking. More than half of ACS patients (59.3\%) were smokers with a duration of more than 10 years. Hypertension is the second most prevalent risk factor after smoking (50.9\%) (Table 1). 497 (34\%) patients had 3 or more risk factors. 761 (52\%) patients had 2 risk factors, and the rest (14\%) only had 1 risk factor.

Of the total patients, 1197 patients (84.1\%) lived during hospitalization, and
228 (15.9\%) patients died in the hospital. The causes of hospital mortality in ACS patients include cardiogenic shock (59.9\%), ventricular arrhythmias (25\%), respiratory failure (6.8\%) and septic shock (5.6\%) (Figure 1). Annual mortality rates from 2014 to 2018 was decreased, from $20.7 \%$, then $20.5 \%, 14.9 \%$ to the last $11.8 \%$ in 2018 (Figure 2). This showed an increase of survival in ACS patients at Dr. Moewardi hospital from 2014 to 2018.

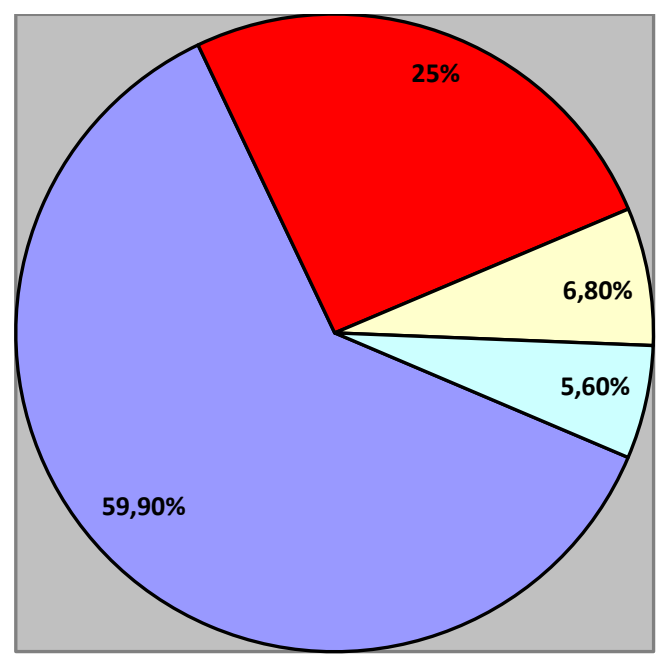

口Cardiogenic Shock

$\square$ Ventricle Arrhytmia

$\square$ Respiratory Failure

$\square$ Septic Shock

Figure 1. Causes of In-Hospital Mortality in ACS patients 


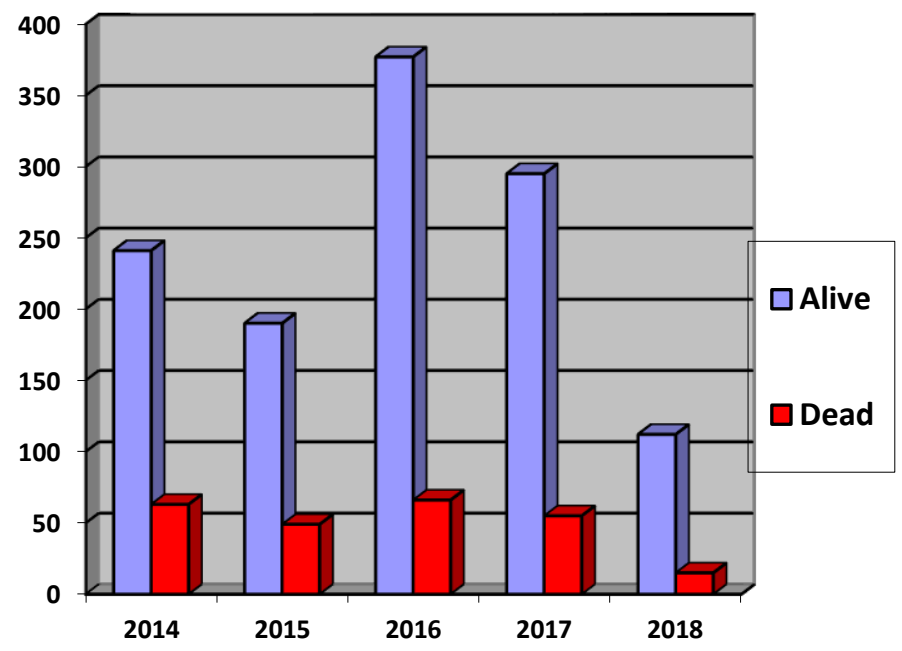

Figure 2. Hospital Mortality Rate for ACS patients from January 2014 to July 2018

Table 2. Sample characteristics

\begin{tabular}{lcccc}
\hline \multirow{2}{*}{ Variable } & All Samples & \multicolumn{2}{c}{ In-Hospital Mortality } & p \\
\cline { 2 - 4 } & & Survived & Dead & \\
\hline Age (years) & $60,00(23-100)$ & $59,46(23-92)$ & $63.39(32-100)$ & 0.001 \\
Men (\%) & $1081(73.9 \%)$ & $925(85.5 \%)$ & $156(14.4 \%)$ & 0.110 \\
Diabetes Mellitus & $395(26.9 \%)$ & $82(20.7 \%)$ & $313(79.2 \%)$ & 0.001 \\
Hypertension & $746(50.9 \%)$ & $637(85.3 \%)$ & $109(14.6 \%)$ & 0.179 \\
History of CAD & $178(12.1 \%)$ & $20(11.2 \%)$ & $158(88.7 \%)$ & 0.040 \\
Smoking & $866(59.3 \%)$ & $737(85.1 \%)$ & $129(14.8 \%)$ & 0.215 \\
Dyslipidemia & $311(21.3 \%)$ & $159(51.1 \%)$ & $152(48.9 \%)$ & 0.132 \\
Type of ACS & & & & \\
STEMI & $922(63.0 \%)$ & $745(80.8 \%)$ & $177(19.2 \%)$ & \\
NSTEMI & $294(20.8 \%)$ & $148(84.4 \%)$ & $46(15.6 \%)$ & \\
UAP & $237(16.2 \%)$ & $237(96.3 \%)$ & $9(3.7 \%)$ & \\
Killip $\geq$ II & $399(27.3 \%)$ & $157(39.3 \%)$ & $242(60.6 \%)$ & 0.001 \\
Hemoglobin & $13.07 \pm 1.89$ & $13.17 \pm 1.79$ & $12.58 \pm 2.28$ & $0.066^{*}$ \\
Leukocyte & $11.62 \pm 4.37$ & $11.14 \pm 4.07$ & $14.11 \pm 4.96$ & $0.021^{*}$ \\
Thrombocyte & $236.00(33-784)$ & $241.44(33-652)$ & $250.49(124-784)$ & $0.309^{* *}$ \\
BUN & $32(13-253)$ & $35(13-57)$ & $60(47-253)$ & $0.001^{* *}$ \\
Creatinine & $1.10(0.72-6.91)$ & $1.18(0.72-2.31)$ & $1.62(1.12-6.91)$ & $0.001^{* *}$ \\
Natrium & $135 \pm 4.68$ & $135 \pm 4.50$ & $134 \pm 5.33$ & $0.099^{*}$ \\
Kalium & $3.9 \pm 0.68$ & $3.9 \pm 0.67$ & $4.1 \pm 0.73$ & $0.106^{*}$ \\
Chloride & $104 \pm 5.38$ & $104 \pm 5.40$ & $103 \pm 5.25$ & $0.266^{*}$ \\
Glucose & $137(45-681)$ & $161(56-681)$ & $221(45-551)$ & $0.001^{* *}$ \\
\hline
\end{tabular}

Table 2 illustrates the relationship between clinical profile and biochemical characteristics of patients with in-hospital mortality. Patients with old age and patients with Killip II presentation had a significant association with in-hospital mortality 
$(\mathrm{p}=$ o.001). ACS patients who had a history of DM and previous CAD had a tendency to dead that were statistically significant $(\mathrm{p}=$ 0.002 and $p=0.042$ ). ACS patients with co-existent cardiovascular risk factor as smoking and dyslipidemia did not reveal any statistically significant higher mortality risk when compared to those without these risk factors.

\section{Relationship between BUN and Creatinine with In-hospital Mortality}

From table 2, it can be seen that hospital deaths occur more in patients with BUN and creatinine levels at the time of initial $\operatorname{admission}(\mathrm{p}=0.001$ and $\mathrm{p}=0.001)$.

Because of BUN and creatinine data are not normally distributed, the data are analyzed using the Mann-Whitney test. Furthermore, we also changed the BUN and creatinine data into categorical data, with BUN cut-off $>50 \mathrm{mg} / \mathrm{dL}$ and creatinine $>1.3 \mathrm{mg} / \mathrm{dL}$. A value below the cut-off is defined as a normal value. The hospital mortality risk between normal and high values at urea and creatinine levels was analyzed by chi-square. Then analyzed by a multiple logistic regression (Table 3).

Table 3. In-hospital Mortality Risk Analysis

\begin{tabular}{|c|c|c|c|c|c|c|c|}
\hline \multirow{2}{*}{$\begin{array}{l}\text { Varia- } \\
\text { bles }\end{array}$} & \multicolumn{3}{|c|}{ Bivariate (Chi-square) } & \multicolumn{2}{|c|}{$\begin{array}{c}\text { Binary Logistic } \\
\text { Regression }\end{array}$} & \multicolumn{2}{|c|}{$\begin{array}{c}\text { Multiple Logistic } \\
\text { Regression }\end{array}$} \\
\hline & Alive & Dead & $\mathbf{p}$ & OR (CI 95\%) & $\mathbf{p}$ & OR (CI 95\%) & $\mathbf{p}$ \\
\hline \multicolumn{8}{|c|}{ BUN (mg/dL) } \\
\hline $\begin{array}{l}\leq 50 \\
>50\end{array}$ & $\begin{array}{c}1097 \\
133\end{array}$ & $\begin{array}{c}35 \\
197\end{array}$ & 0.001 & $\begin{array}{l}5.01(1.00- \\
7.01)\end{array}$ & 0.001 & \multirow[t]{2}{*}{$\begin{array}{c}13.92(10.09- \\
16.11)\end{array}$} & \multirow[t]{2}{*}{0.001} \\
\hline \multicolumn{6}{|c|}{ Creatinine (mg/dL) } & & \\
\hline $\begin{array}{l}\leq 1.3 \\
>1.3\end{array}$ & $\begin{array}{l}969 \\
261\end{array}$ & $\begin{array}{l}131 \\
101\end{array}$ & \multirow[t]{2}{*}{0.001} & $\begin{array}{c}3.60\left(2.23^{-}\right. \\
5.80)\end{array}$ & \multirow[t]{2}{*}{0.031} & \multirow[t]{2}{*}{$4.24(3.14-5.39)$} & \multirow[t]{2}{*}{0.027} \\
\hline * Adjus & vith le & te, glu & & ip classificatio & & & \\
\hline
\end{tabular}

From the results of logistic regression analysis, high level BUN at the first time of admission had 5 times higher $(\mathrm{OR}=5.01$, 95\% CI 1.00-7.01, $\mathrm{p}=0.001$ ) risk for dying in hospital compared to normal BUN values in patients with ACS. Likewise, with creatinine, patients with high creatinine values, the risk of death in the hospital was 4 times $(\mathrm{OR}=3.60 ; 95 \% \mathrm{CI}=2.23-5.80)$ higher compared to the normal creatinine value ( $\mathrm{p}$ $<0.005$ ). When added with leukocytes and glucose variables in the multivariate analysis with logistic regression, a high level BUN > $50 \mathrm{mg} / \mathrm{dL}$ had a risk of death 14 times compared to normal group (OR= 13.92, 95\% $\mathrm{CI}=10.09-16.11, \quad \mathrm{p}=0.001)$. Increased BUN value has more effect on the risk of in-hospital mortality compared to creatinine values (OR 13.92 vs OR 4.24).

On the ROC curve, BUN correlated significantly with in-hospital mortality, with Area Under Curve (AUC) 87.1\% (P $<0.001$, 95\% CI 0.842-0.899). Compared with creatinine, the AUC value of creatinine was lower, which is $61.2 \%$ ( $95 \% \mathrm{CI}=0.57$ to $0.65 ; \mathrm{p}<0.001$ ) (Figure 3). It can be concluded that BUN are more accurate than creatinine as predictors of in-hospital mortality in patients with ACS. In this curve can also obtained the optimum BUN cut-off value $>50 \mathrm{mg} / \mathrm{dL}$ has sensitivity of $84.9 \%$ and specificity $89.2 \%$ as a predictor of death, and creatinine value $<1.3 \mathrm{mg} / \mathrm{dL}$ has a sensitivity of $59.5 \%$ and specificity of $71.4 \%$. 


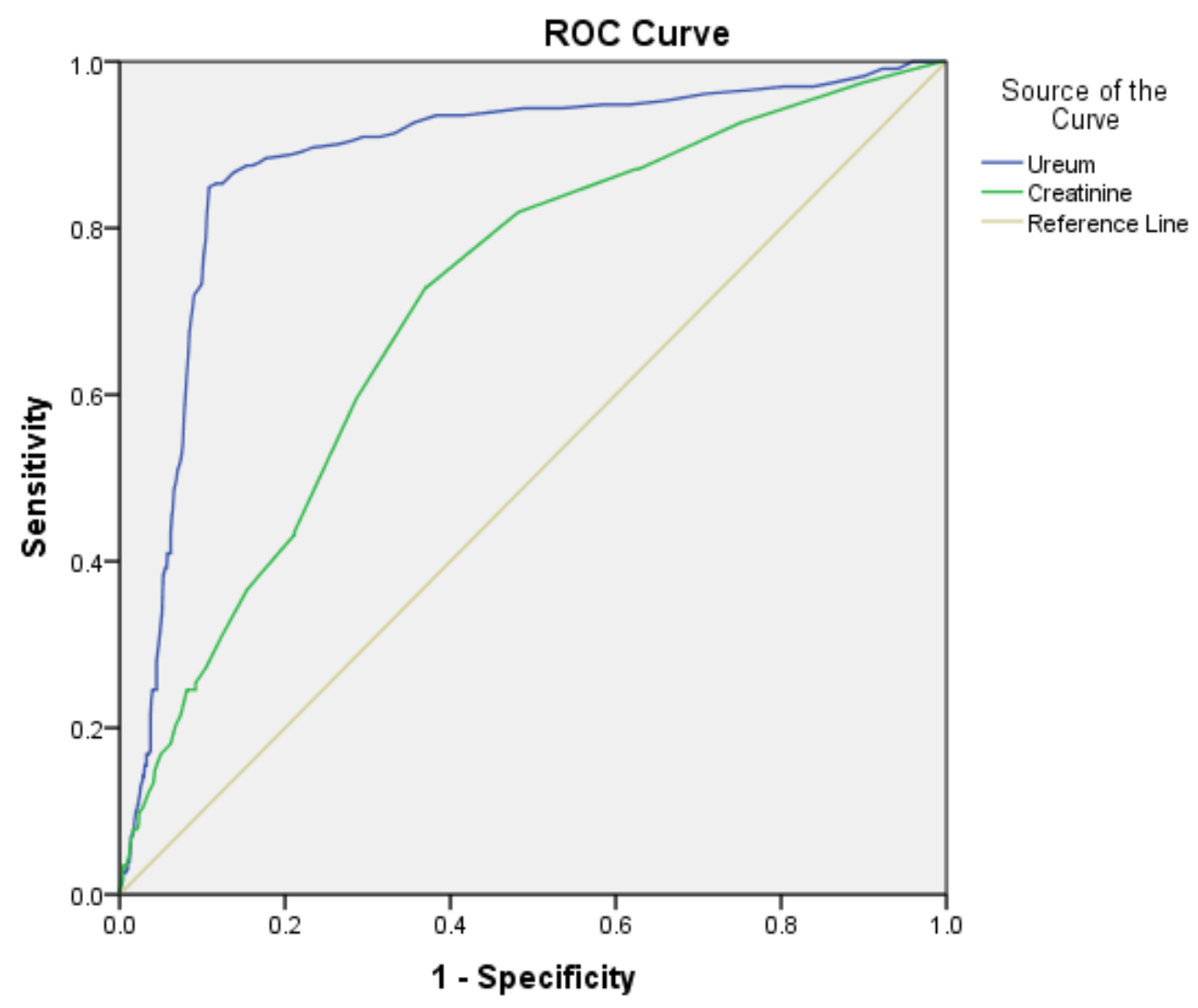

Diagonal segments are produced by ties.

\section{Figure 3. Comparison of BUN and Creatinine as In-hospital Mortality Predictor}

\section{DISCUSSION}

In this study, the majority of patients presented at mean age of 60 years, with the youngest was 23 , and the oldest was 100. A finding which is consistent with previously published reports on CREATE Registry, which report a clear preponderance of male subjects, with average age of $57 \pm 12.1$ (Xavier et al., 2008). The majority of ACS patients in our study were male, similar to ACS registry study that conduct around Jakarta (JAC Registry), where $85 \%$ of ACS patients are dominated by men (Dharma et al., 2016). In addition, most ACS patients in this study were diagnosed with STEMI (62\%). This finding is similar to CREATE registry study (Xavier et al., 2008). In contrast, reports from developed countries indicate that less than $40 \%$ of patients with
\end{abstract}

ACS had STEMI (Ranjith et al., 2011), suggesting that Asian people are more likely to experience extensive myocardial infarction and worse prognosis than other populations (Ravi et al., 2013).

The most commonly observed risk factors in the current study population included smoking, hypertension, and diabetes mellitus, while a history of $\mathrm{CAD}$ and dyslipidemia occurred less frequently. Although the findings presented here are consistent with those of the European and Mediterranean population (Euro Heart Survey) (Mandelzweiget al., 2006),smokers and patients with a history of hypertension in this study have a greater proportion compared to the Euro Heart Survey study (59\% and $51 \%$ vs. $18.5 \%$ and $39 \%$ ). Compared with Canadian subjects in the 
CANRACE registry study, ACS patients in this study had a similar prevalence of diabetes and hypertension ( $27 \%$ and $51 \%$ vs $26 \%$ and $59 \%$ ), but the prevalence of smoking was far more in our study (59\% vs 27\%) (Paul, 2010). Apart from the descripttive explanation above, our study also shows that there is a significant relationship between the increase in BUN and creatinine levels with in-hospital mortality of ACS patients. BUN and creatinine are known as predictor of kidney dysfunction. One condition that can worsen the outcome of acute coronary syndrome is acute heart failure, which can cause acute kidney injury. This condition is called type 1 cardiorenal syndrome (CRS). A number of literature shows that patients with CRS type 1 are at high risk for poor outcomes (Paul, 2010).

This independent effect may be due to the associated acceleration in cardiovascular pathobiology due to renal dysfunction through neurohormonal activation, cell signaling, oxidative stress, or fibrosis cascades. Acute kidney injury is an independent risk factor for 1-year mortality in patients with acute heart failure, including patients with an increased STEMI who experience signs and symptoms of heart failure or have a decreased left ventricular ejection fraction (JoAnn et al., 2011).In previous studies, to determine the presence of renal dysfunction, clinicians used serum creatinine, estimated creatinine clearance, or glomerular filtration rate (GFR). However, estimation of renal function based on serum creatinine is imperfect and may not be sufficient to assess acute changes in actual GFR or other aspects of renal function, especially when GFR is only slightly reduced (Kirtane et al., 2005).

Blood urea nitrogen concentrations have been considered as more specific markers of kidney function than serum creatinine and GFR estimates. In addition, an increase in urea is independent, not solely due to a decrease in GFR. This is because urea reabsorption is a passive process associated with sodium and water reabsorption due to activation of the sympathetic nervous system and Renin Angiotensin Aldosterone System (RAAS), where the condition better represents the kidney response to systemic hypoperfusion due to a decrease in cardiac output rather than intrinsic renal dysfunction (Hata et al., 2010). In this context, the urea level at the time of initial hospitalization and its increase during hospitalization can be a simple marker of hemodynamic deterioration, cardiorenal dysfunction and neurohormonal activation (Horiuchi et al., 2018).

Unlike creatinine, elevated urea levels are associated with adverse outcome prognosis regardless of age, SBP, heart rate, Killip class, troponin levels, type B natriuretic peptides and ST segment changes in patients with acute heart disease such as ACS, acute heart failure and post Coronary Artery Bypass Graft (CABG) (Cauthen et al., 2008). According to a study by Cauthen and friends, the increased risk of death within 1 year after myocardial infarction was associated with BUN levels $>6.1 \mathrm{mmol} /$ $\mathrm{L}$, and this marker was more important for prognosis of MACE than serum creatinine or creatinine clearance (Aronson et al., 2008). Aronson and friends also stated that an increase in urea levels was a significant predictor of mortality in ACS patients $>65$ years. The opinions of some of these researchers are consistent with the results of our study that high urea levels are independent risk factors for hospital mortality in ACS patients. The level of BUN can add prognosis information to evaluating ACS patients at the time of initial inpatient care which is very useful for patient management 
This study has several limitations. This study is a cohort retrospective, involving all ACS patients from January 2013 to July 2018 in Dr. Moewardi hospital which is a type A referral hospital for heart disease in the south region of Central Java. So the results of this study are quite representative for ACS patients in midurban city of Indonesia.

Elevated BUN and creatinine are independent predictors of in-hospital mortality in ACS patients. A high level BUN at admission could be a more accurate predictor of in-hospital mortality than creatinine. Serum BUN may serve a simple marker to identify ACS patients at high risk.

\section{AUTHOR CONTRIBUTIONS}

Trisulo Wasyanto and Annisa Tridamayanti collected the data, measured creatinine level, did data analysis, and wrote the manuscript.

\section{CONFLICT OF INTEREST}

The authors declare there is no conflict of interest.

\section{ACKNOWLEDMENT}

We would like to thank to Dr. Moewardi Hospital that give permission to collect the data.

\section{FUNDING AND SPONSORSHIP}

No funding and sponsorship.

\section{REFERENCE}

American Diabetes Association (2004). Diagnosis and Classification of Diabetes Mellitus. Diabetes Care; 27 (1): s5-s10.

Aronson D, Hammerman H, Beyar R, et al. 2008. Serum blood urea nitrogen and long-term mortality in acute ST- elevation myocardial infarction. Int $\mathrm{J}$ Cardiol; 3(127):380-385.

Aronson D, Mittleman MA, Burger AJ, et al. (2004). Elevated blood urea nitrogen level as a predictor of mortality in patients admitted for decompensated Heart Failure. Am J Med; 116:466473 .

Catapano AL, Graham I, Backer GD, et al. (2016). ESC/EAS Guidelines for the Management of Dyslipidemias. Eur Heart J; 37:2999-3058

Cauthen CA, Lipinski MJ, Abbate A, et al. (2008). Relation of blood urea nitrogen to long-term mortality in patients with heart failure. Am J Cardiol; 101: 1643-7.

Chen CY, Yoshida A, Asakura M, et al. 2010. Serum blood urea nitrogen and plasma brain natriuretic Peptide and low diastolic blood pressure predict Cardiovascular morbidity and mortality following discharge in acute decompensated heart failure patients. Circulation J; 76:2372-2379.

Dharma S, Andriantoro H, Purnawan I, et al. (2016). Characteristics, Treatment and In-Hospital Outcomes of patients with STEMI in a Metropolitan Area of a Developing Country: An Initial Report of the Extended Jakarta Acute Coronary Syndrome registry. BMJ Open; 6: e012193.

Go AS, Mozaffarian D, Roger VL, et al. (2013). Heart disease and stroke statistics-2013 update: a report from the American Heart Association. Circulation; 127:236-245.

Gibson CM, Pinto DS, Murphy SA, et al. (2003). Association of creatinine and creatinine clearance on presentation in acute myocardial infarction with subsequent mortality. J Am Coll Cardiol; 42: 1535-154. 
Granger CB, Goldberg RJ, Dabbous OM, et al. (2003). Predictors of hospital mortality in the Global Registry of Acute Coronary Events. Arch Intern Med; 163:2345-53.

Grundy SM, Stone NJ, Bailey AL, et al. (2018). ACC/AHA/AACVPR/AAPA/ABC/ACPM/ADA/AGS/ APhA/ASPC/NLA/PCNA guideline on the management of blood cholesterol: A report of the American College of Cardiology Foundation/American Heart Association Task Force on Clinical Practice Guidelines. J Am Coll Cardiol. 10: 104-113.

Hata N, Yokoyama S, Shinada S, et al. (2010). Acute kidney injury and outcomes in acute decompensated heart failure: Evaluation of the RIFLE criteria in an acutely ill heart failure population. Eur J Heart Fail (12): 32-37.

Horiuchi Y, Aoki J, Tanabe K, et al. (2018). A high level of blood urea nitrogen is a significant predictor for in-hospital mortality in patients with acute myocardial infarction. Int Heart $\mathrm{J}$; 59(2): 263-271.

JoAnn L, Robert WS (2011). Blood urea nitrogen a marker for adverse effects of loop diuretics. JACC; 58(4): 122131.

Kazory A (2010). Emergence of blood urea nitrogen as a biomarker of neurohormonal activation in heart failure. Am J Cardiol; 106:694-700.

Kimura K, Morita H, Daimon M, et al. (2016). Utility of cystatin C for estimating glomerular filtration rate in patients with muscular dystrophy. Int Heart J; 57:386-388.

Kirtane AJ, Leder DM, Waikar SS, et al. (2005). Serum blood urea nitrogen as an independent marker of subsequent mortality among patients with acute coronary syndromes and normal to mildly reduced glomerular filtration rates. J Am Coll Cardiol; 45:1781-6.

Lee PT, Chao TH, Huang YL, et al. (2016). Analysis of the clinical characteristics, management, and causes of death in patients with ST-segment elevation myocardial infarction treated with primary percutaneous coronary intervention from 2005 to 2014. Int Heart $\mathrm{J} ; 57: 541-6$.

Mandelzweig L, Battler A, Boyko V, et al. (2006). Euro Heart Survey Investigators. The second Euro Heart Survey on acute coronary syndromes: Characteristics, treatment, and outcome of patients with ACS in Europe and the Mediterranean Basin in 2004. Eur Heart J; 27:2260-2262.

Paul AM (2010). Cardiorenal Syndromes: Pathophysiology to Prevention. Int J Nephrol(12): 762-890.

Ranjith N, Pegoraro RJ, Zaahl MG (2011). Risk factors associated with acute coronary syndromes in South African Asian Indian Patients [The AIR Study]. J Clinic Experiment Cardiol; 2:163.

Ravi RB, Shaun GG, Raymond TY et al. (2013). Treatment and outcomes of patients with suspected acute coronary syndromes in relation to initial diagnostic impressions (Insights from the Canadian Global Registry of Acute Coronary Events [GRACE] and Canadian Registry of Acute Coronary Events [CANRACE]). J AM Card; 111(2): 202-207.

Shiraishi J, Nakamura T, Shikuma A, et al. (2016). Relationship between mean blood pressure at admission and inhospital outcome after primary percutaneous coronary intervention for acute myocardial infarction. Int Heart J; 57: 547-52. 
Thygesen K, Alpert JS, Jaffe AS, et al. (2019). Fourth universal definition of myocardial infarction. Eur Heart J; 40(3):237-269.

Xavier D, Pais Prem, Devereaux PJ, et al. (2008). On behalf of the CREATE registry investigators. Treatment and outcomes of acute coronary syndromes in India [CREATE]: a prospective analysis of registry data. Lancet; 371:1435-1442. 